Isabela Vieira ${ }^{1}$

\author{
${ }^{1}$ Médica psiquiatra; Mestre em Psiquia- \\ tria pelo Programa de Pós-Graduação \\ em Psiquiatria e Saúde Mental do \\ Instituto de Psiquiatria da Universidade \\ Federal do Rio de Janeiro (UFRJ). \\ *Trabalho baseado na Dissertação de \\ Mestrado intitulada Utilidade clínica do \\ conceito de burnout: revisão sistemática de \\ estudos longitudinais, apresentada em \\ 2009 no Instituto de Psiquiatria (IPUB) \\ da Universidade Federal do Rio de \\ Janeiro (UFRJ). \\ Contato: \\ Rua Dois de Dezembro, 38 - sala 909 \\ - Flamengo \\ CEP: 22220-040, Rio de Janeiro-RJ \\ E-mail: \\ isabelamvieira@gmail.com
}

\section{Conceito(s) de burnout: questões atuais da pesquisa e a contribuição da clínica*}

\author{
Concept(s) on burnout - current themes in research and the \\ contribution of clinical practice
}

\section{Resumo}

Considerando o crescente interesse científico no fenômeno burnout, apresentamos uma amostra da diversidade teórica que forma o campo de pesquisa neste tema a partir de algumas questões atuais de discussão sobre o seu conceito. Discutimos aparentes limitações da abordagem empírica à compreensão da natureza conceitual do burnout, apontando uma possível contribuição da clínica (dentro do campo da Psiquiatria) a este debate.

Palavras-chave: burnout - conceito; estresse mental; psicologia organizacional; fadiga nervosa; estresse ocupacional.

\begin{abstract}
Considering the growing scientific interest in burnout phenomenon, we present a sample of the theoretic diversity that constitutes the research field in this area presenting some of the debates currently being held on its concept. We discuss apparent limitations of the empirical approach on the comprehension of burnout conceptual nature, indicating a possible contribution from the clinical practice (within the field of psychiatry) to this debate.
\end{abstract}

Keywords: burnout - concept; mental stress; organizational psychology; nervous fatigue; occupational stress. 


\section{Introdução}

O termo burnout significa "queima" ou "combustão total”. Faz parte do vocabulário coloquial em países de língua inglesa e costuma ser empregado para denotar um estado de esgotamento completo da energia individual associado a uma intensa frustração com o trabalho (MASLACH; SCHAUFELI; LEITER, 2001).

Este fenômeno passou a ser objeto de estudo científico na década de 1970 com a primeira descrição clínica feita por Freudenberger (1974). Desde então, vem despertando o interesse dos pesquisadores, especialmente no campo da Saúde Ocupacional. Isto pode ser verificado pelo volume crescente de publicações sobre o tema (Gráfico 1). Em 2009, foram indexados, na base ISI Web of Science, 208 artigos (em inglês) contendo burnout no título.

O conhecimento derivado da pesquisa empírica a respeito do burnout pode ser resumido como se segue.

Em geral, ele é definido como uma reação negativa ao estresse crônico no trabalho (SHIROM, 2003; HONKONEN et al., 2006; AHOLA et al., 2006a). Manifesta-se basicamente por sintomas de fadiga persistente, falta de energia, adoção de condutas de distanciamento afetivo, insensibilidade, indiferença ou irritabilidade relacionadas ao trabalho de uma forma ampla, além de sentimentos de ineficiência e baixa realização pessoal. Trata-se de uma condição crônica (SHIROM, 2003), determinada principalmente por fatores da organização do trabalho, tais como sobrecarga, falta de autonomia e de suporte social para a realização das tarefas (MASLACH; SCHAUFELI; LEITER, 2001; SCHAUFELI; ENZMANN, 1998). A chamada reestruturação produtiva e as demissões em massa também são apontadas como fatores de risco (KALIMO, 2000). Traços de personalidade teriam menor peso para o desencadeamento do quadro (MASLACH; SCHAUFELI; LEITER, 2001). O burnout está ainda associado a consequências negativas, dentre as quais:
- No nível socioeconômico: absenteísmo, queda de produtividade (PARKER; KULIK, 1995) e aposentadoria precoce (WEBER; WELTLE; LEDERER, 2005);

- Para a saúde física: aumento do risco cardiovascular (HONKONEN et al., 2006; MELAMED et al., 2006a), alterações fisiológicas e metabólicas como desregulação do eixo hipotálamo-hipófise-adrenais (GROSSI et al., 2005), diabetes tipo 2 (MELAMED et al., 2006a), elevação de lipídios séricos (SHIROM et al., 1997) e alterações do sistema imune (LEHRMAN et al., 1999), além de distúrbios musculoesqueléticos (HONKONEN et al., 2006);

- Para a saúde mental: associação com ansiedade e, em especial, depressão (MASLACH; SCHAUFELI; LEITER, 2001; AHOLA et al., 2005), além de abuso de álcool (AHOLA et al., 2006b).

Tal conjunto de informações provém majoritariamente de estudos transversais. Destacam-se os estudos de prevalência em amostras de trabalhadores das mais diversas categorias profissionais. Esta prevalência varia em função da população estudada e da metodologia utilizada, oscilando entre aproximadamente $10 \%$ a taxas superiores a 30\% (WEBER; JAEKEL-REINHARD, 2000). Em estudos com amostras representativas da população geral ativa, a taxa de burnout variou entre 5\% e 7\% (KALIMO, 2000; HALLSTEN, 2005). A maior parte dos trabalhos publicados utiliza a definição de burnout proposta por Maslach (que consiste na tríade Exaustão, Despersonalização/Cinismo e baixa Realização Pessoal/Ineficácia - que comentaremos a seguir). Há, no entanto, uma tendência de crescimento do número de estudos longitudinais (TOPPINEN-TANNER et al., 2005; MOMMERSTEEG et al., 2006; MELAMED et al., 2006b; MOCH et al., 2003), especialmente a partir do ano 2000, o que tem possibilitado uma melhor investigação das relações entre burnout e os possíveis fatores de risco e consequências apontados pelos estudos transversais.
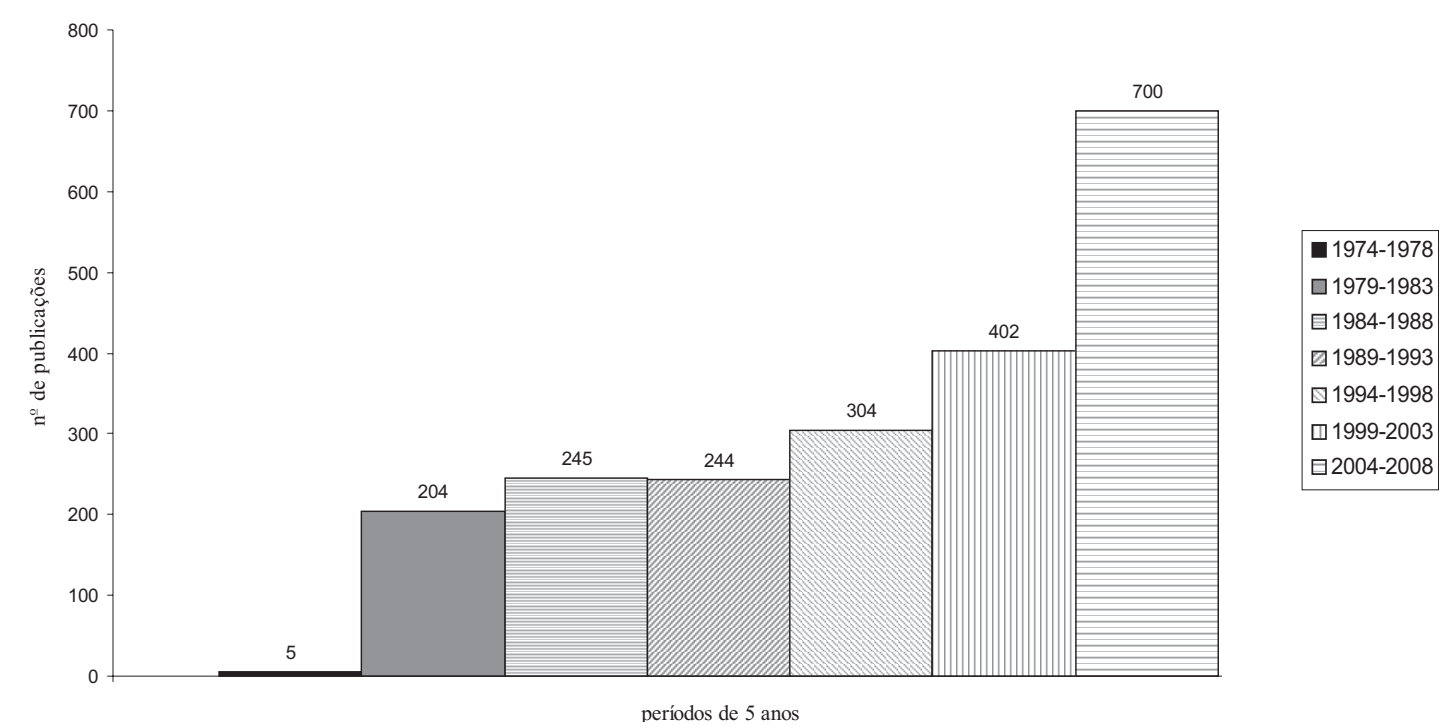

Gráfico 1 Número de publicações com burnout no título indexados na base ISI Web of Science, por períodos de 5 anos, de 1974 a 2008 
No Brasil, apesar do interesse científico crescente, ainda há poucas publicações sobre burnout (uma busca por artigos com burnout no título, publicados em português e indexados na base LILACS, encontrou 54 estudos desde 1987), a maior parte delas voltadas para a detecção de taxas de prevalência, principalmente entre profissionais de saúde e da educação. A quase totalidade dos trabalhos publicados utiliza o conceito de Maslach (CARLOTTO; PALAZZO, 2006; SILVEIRA et al., 2005; TUCUNDUVA et al., 2006).

O conhecimento produzido até o momento, quando visto superficialmente, dá a impressão de ser bastante homogêneo. Principalmente o fato de haver uma definição dominante de burnout reforça esta impressão. Porém, se nos aprofundamos um pouco, percebemos logo que o debate científico é intenso e vem gerando perspectivas teóricas variadas.

O presente artigo pretende apresentar um pouco desta diversidade teórica, enfocando, nesta oportunidade, alguns aspectos da discussão atual sobre o conceito de burnout. A partir destes aspectos, discutiremos os limites ou os impasses da pesquisa empírica neste campo de investigação, pensando em uma possível contribuição da clínica à resolução destes impasses.

\section{Um conceito em construção}

O estudo sistemático do burnout inaugura-se em 1974 a partir da descrição clínica, pelo psicanalista norte-americano Herbert Freudenberger, de um quadro de esgotamento físico e mental com intensa irritabilidade relacionado a condições adversas de trabalho de profissionais de saúde atuando na área de dependência química (FREUDENBERGER, 1974; FREUDENBERGER; RICHELSON, 1987). Em sua definição, burnout é um “incêndio interno", um "esgotamento dos recursos físicos e mentais"; é "esgotar-se para atingir uma meta irrealizável” imposta pelo próprio indivíduo ou pela sociedade. Para ele, tal esgotamento vai ocorrer na área da vida onde há mais expectativa de sucesso - em geral, no trabalho (FREUDENBERGER; RICHELSON, 1987).

Paralelamente, a psicóloga social Christina Maslach (e colaboradores), pesquisando trabalhadores dos setores de serviços/cuidados (os chamados human services), também dá o nome de burnout a um fenômeno semelhante, definindo-o como uma "síndrome psicológica em reação a estressores interpessoais crônicos no trabalho" e identificando nele três componentes principais (MASLACH; SCHAUFELI; LEITER, 2001):

- Exaustão emocional: caracterizada por cansaço extremo e sensação de não ter energia para enfrentar o dia de trabalho;
- Despersonalização: adoção de atitude de insensibilidade ou hostilidade em relação às pessoas que devem receber o serviço/cuidado;

- Perda da realização pessoal: sentimentos de incompetência e de frustração pessoal e profissional.

A criação de um instrumento de aferição (Maslach Burnout Inventory - MBI [MASLACH; JACKSON; LEITER, 1996]) viabiliza o estudo epidemiológico do burnout e, rapidamente, o conceito de Maslach torna-se o mais conhecido e adotado. Na prática, a tríade Exaustão, Despersonalização e baixa Realização Pessoal tornase sinônimo de burnout.

Apesar de o MBI ter sido criado para detectar burnout no âmbito das relações entre profissionais provedores de serviços/cuidados e seus receptores (daí a nomenclatura original MBI-HSS - Human Services Survey), vários pesquisadores passaram a aplicá-lo em diversos outros grupos profissionais não especificamente pertencentes aos chamados human services (MASLACH; JACKSON; LEITER, 1997). Com isso, constatou-se que a diferenciação entre as dimensões do MBI sofria alterações em comparação aos resultados obtidos com as amostras de trabalhadores dos human services. Por este motivo, houve a necessidade de adaptar o instrumento de maneira a manter seus parâmetros de validade. Surge, então, a versão para educadores (MBI-ES - Education Survey) e, posteriormente, o MBI-GS (General Survey) (MASLACH; JACKSON; LEITER, 1997).

O objetivo do MBI-GS, portanto, é detectar problemas no relacionamento das pessoas com o trabalho (não necessariamente problemas com outras pessoas no ambiente de trabalho). As adaptações ao instrumento original consistiram na renomeação das dimensões Exaustão Emocional (para Exaustão), Despersonalização (para Cinismo/Ceticismo) ${ }^{2}$ e baixa Realização Pessoal (para Eficácia Pessoal) e na redução do número de sentenças.

A dimensão Exaustão é mais genérica, sem a ênfase na reação emocional; a dimensão Cinismo passa a refletir indiferença ou atitude distanciada em relação ao trabalho (e não em relação a relacionamentos interpessoais no trabalho, como na Despersonalização); e a dimensão Eficácia Pessoal abrange aspectos sociais e não sociais da realização profissional, além de enfocar mais as expectativas individuais de rendimento do que a dimensão Realização Pessoal (MASLACH; JACKSON; LEITER, 1997).

Apesar das adaptações visando a uma melhor apreensão do fenômeno, alguns pesquisadores (SHIROM, 2003; KRISTENSEN et al., 2005) detectam falhas na concepção de Maslach, tanto teóricas (na elaboração do conceito), quanto metodológicas (na elaboração do MBI), pondo em risco a validade dos achados epidemiológicos. Baseados em mode-

\footnotetext{
${ }^{2} \mathrm{O}$ termo original em inglês, Cynicism, foi traduzido aqui como Cinismo ou Ceticismo. A noção de Ceticismo, embora um pouco diferente da de Cinismo, foi pensada como uma opção válida para tradução, entre outros motivos, por amenizar certa conotação pejorativa que o termo "cinismo" possui em nosso idioma.
} 
los teóricos e em dados empíricos, eles propõem novas definições de burnout com o objetivo de aprimorar o conceito. Destacamos aqui os conceitos de Pines e Aronson, Shirom e Melamed, Demerouti e colegas e Kristensen e colegas.

- Pines e Aronson: definiram burnout como "um estado de exaustão física, emocional e mental causado por um envolvimento de longo prazo em situações de alta demanda” (PINES; ARONSON, 1981; SCHAUFELI et al., 2001). Não restringem o conceito ao campo ocupacional (poderia ocorrer na vida conjugal, por exemplo). A síndrome resultante consistiria em sentimentos de desamparo, desesperança, de estar preso numa armadilha, pouco entusiasmo, irritabilidade, além do cansaço físico e emocional e de baixa autoestima (PINES; ARONSON, 1981; SHIROM, 2003).

Seu referencial teórico vem da perspectiva existencial. De acordo com esta visão, a raiz do problema estaria na necessidade das pessoas de atribuir um significado para suas vidas como uma forma de diminuir a angústia provocada pela consciência da morte; assim, elas atribuem também, ao trabalho, um sentido especial, levando à sua idealização. Então, quando percebem que seu trabalho não é mais capaz de preencher tais expectativas, advêm sentimentos de fracasso, tristeza e, eventualmente, o burnout (PINES; KEINAN, 2005).

O instrumento desenvolvido por Pines e Aronson, chamado Burnout Measure (BM), é apontado como o segundo mais utilizado, até 1998, após o MBI (em 5\% dos estudos, segundo Schaufeli e Enzmann [1998]). Entretanto, atualmente vem caindo em desuso devido a limitações metodológicas importantes na sua elaboração (HALBESLEBEN; DEMEROUTI, 2005; ENZMANN et al., 1998).

- Shirom e Melamed: em sua definição, burnout é um estado afetivo singular caracterizado pela sensação de perda de energia física, mental e cognitiva, que ocorre como reação ao estresse crônico (SHIROM, 1989; 2003). Apesar de não mencionar especificamente o contexto laboral na definição, na prática tem sido considerado como condição relacionada ao trabalho (em função de o estresse ocupacional ser uma das principais fontes de estresse crônico). Suas três dimensões são denominadas Fadiga Física (sensação de cansaço e baixa energia na execução de tarefas cotidianas, como, por exemplo, acordar pela manhã para ir ao trabalho), Exaustão Emocional (sensação de estar fraco demais para ter empatia com clientes ou colegas e não ter a energia necessária para investir em relacionamentos interpessoais no trabalho) e Desgaste Cognitivo (sensação de raciocínio lento e baixa agilidade mental); cada componente abrange um domínio particular da depleção energética (SHIROM et al., 2005).

Esta concepção de burnout baseia-se na teoria da Conservação de Recursos (Conservation of Resources - COR). Segundo esta teoria, estresse é definido como qualquer situação de ameaça ou perda efetiva de recursos energé- ticos, à qual o organismo se contrapõe ativamente (o que acarreta também um gasto adicional de energia), eventualmente levando a um fluxo contínuo de perda - manifestado pelo burnout (SHIROM et al., 2005).

O instrumento desenvolvido pelos autores é o Shirom-Melamed Burnout Measure - SMBM (HOBFOLL; SHIROM, 1993).

- Demerouti e colegas: para estes autores, o burnout é composto de apenas duas dimensões: Exaustão e Desengajamento (análoga à Despersonalização). Esta última representaria uma reação de rejeição emocional, cognitiva e comportamental ao trabalho (visto como desinteressante, não desafiador e "aversivo") em termos de uma desilusão (DEMEROUTI et al., 2002a). Para os autores, a Despersonalização seria não mais que uma forma de Desengajamento (DEMEROUTI et al., 2002b). Desenvolveram o Oldenburg Burnout Inventory (OLBI), que abrange os aspectos físicos e cognitivo da exaustão (em contraposição ao MBI, que apreenderia apenas o aspecto afetivo da exaustão). Este instrumento também representaria um aprimoramento em relação ao MBI quanto à sua estrutura (por exemplo, a formulação das sentenças). Como Maslach, Schaufeli e Leiter (2001), consideram o burnout uma reação ao estresse ocupacional, não restrito a profissões específicas (HALBESLEBEN; DEMEROUTI, 2005).

- Kristensen e colegas: consideram como aspecto central da síndrome a fadiga/exaustão e a sua atribuição, pelo indivíduo, à determinada esfera da sua vida. Desta forma, o instrumento desenvolvido pelos autores - o Copenhagen Burnout Inventory (CBI) - avalia burnout em três áreas: pessoal (genérico, isto é, representando o quanto o indivíduo se sente cansado ou exausto, de maneira geral), ligado ao trabalho (o grau de exaustão física ou psicológica que é percebida pelo sujeito como relacionada ao trabalho) e ligado ao cliente (ou seja, o quanto a exaustão da pessoa é atribuída ao seu trabalho com outras pessoas; o termo "cliente" pode ser substituído por "pacientes”, "colegas", "filhos", "estudantes" etc., dependendo do contexto). Desta forma, fica evidente que esses autores não consideram o burnout como fenômeno exclusivamente relacionado à atividade profissional (KRISTENSEN et al., 2005).

Vê-se, portanto, que o conceito de burnout está em franco processo de revisão crítica, em construção.

\section{Limites da pesquisa e a contribuição da clínica: a questão subjetiva}

Em um volume do periódico Work \& Stress dedicado exclusivamente ao burnout, conclui-se pela necessidade urgente de estabelecer de maneira mais precisa o seu conceito (COX; TISSERAND; TARIS, 2005). Aqui destacamos duas questões importantes no debate científico atual sobre o conceito de burnout, propostas naquela publicação: 
- Burnout se resume à dimensão Exaustão?

- Burnout se dá especificamente no contexto do trabalho?

A primeira questão refere-se à discussão sobre a uni ou a multidimensionalidade do conceito de burnout. Baseia-se no relativo consenso, entre os pesquisadores, em se considerar a característica da exaustão como o núcleo do fenômeno. A preponderância deste componente tem levado alguns autores a indagar se não seria o caso de restringir o conceito de burnout a esta dimensão. $\mathrm{O}$ argumento proposto é o de que, pelo fato de cada dimensão associar-se a diferentes antecedentes e consequências, elas deveriam ser consideradas como constructos diversos, não havendo razão para reuni-las sob uma única rubrica (SHIROM, 2003).

Há também pesquisadores que preferem classificar como burnout apenas os casos em que ocorre a presença conjunta das dimensões Exaustão e da Despersonalização - ou Cinismo/Ceticismo (SONNENTAG, 2005). A questão do debate, então, desloca-se um pouco para a dimensão da Despersonalização/Cinismo (DE/C): ela faria mesmo parte do fenômeno?

A associação entre Exaustão e Despersonalização/ Cinismo é forte, conforme demonstram vários estudos (LEITER, 1989; MASLACH; SCHAUFELI; LEITER, 2001). Alguns autores entendem que a DE, enquanto estratégia de coping (mecanismo de defesa de tipo comportamental), seria o caminho "natural" contra a Exaustão. O simples fato de se constituir numa estratégia de coping já seria, por si só, motivo para sua exclusão do conceito (KRISTENSEN et al., 2005; SHIROM, 2003).

No entanto, outros estudiosos argumentam que a DE/C não só deveria permanecer integrada ao conceito de burnout, como seria, inclusive, a sua dimensão mais específica, já que, sem ela, não haveria como diferenciar o burnout de um quadro de fadiga prolongada (SONNENTAG, 2005). Leiter (1989) já apontava o caráter disfuncional (ou seja, "patológico") da DE: caso fosse uma defesa "saudável”, sua associação com a Exaustão seria negativa, e não positiva. Schaufeli e Taris (2005) observam, ainda, que não há incompatibilidade em se considerar uma estratégia de coping parte de uma síndrome, apenas por se tratar de um mecanismo comportamental. Tomam como exemplo a síndrome ansiosa: esta compõe-se de sintomas físicos, psíquicos e também pela adoção de condutas de evitação fóbica. A evitação é um sintoma comportamental, e nem por isso deixa de fazer parte da síndrome.

Por outro lado, a baixa Realização Pessoal/Eficácia tende a ser deixada de lado como componente da síndrome de burnout, uma vez que se associa de maneira muito diferente das demais dimensões a antecedentes e a consequências. Poderia ser considerada como mais uma consequência de burnout (DEMEROUTI et al., 2002b).

A segunda questão - se o burnout é estritamente relacionado ao trabalho ou pode ocorrer em outras áreas da vida - justifica-se com base no seguinte raciocínio: considerando-se que o burnout consiste numa resposta ao estresse crônico, não importaria, na verdade, o contexto deste estresse crônico. Esta linha de pensamento prioriza a resposta fisiológica de estresse; porém, paradoxalmente, como consequência, temos que, quanto mais abrangente a definição de burnout (quanto menos ligado ao trabalho), mais próximo ao conceito de fadiga ele se torna (COX; TISSERAND; TARIS, 2005).

Contrários a esta perspectiva, Schaufeli e Taris (2005) apontam a importância de manter o conceito como associado, se não ao trabalho stricto sensu, a atividades análogas ao trabalho, definidas como todas aquelas atividades estruturadas, de natureza coercitiva, e dirigidas a objetivos específicos. Ou seja, atividades que, de um ponto de vista psicológico, são similares ao trabalho (por exemplo: estudo, cuidar de familiares doentes, treinamento de atletas).

À primeira vista, tais pontos de discussão podem parecer meramente teóricos, bastando tomar uma decisão arbitrária para resolvê-los. Porém, na experiência de atendimento com os pacientes, estas são questões que se colocam prontamente; mais: elas se impõem. Vejamos de que forma.

O que mais chama a atenção, em nossa prática clínica com pacientes que podem ser classificados como portadores de burnout, é o seu relato recorrente de sentimentos negativos como desencanto e profunda desilusão, chegando à adoção de condutas de evitação ou de verdadeira aversão a tudo o que remeta ao trabalho. Por exemplo: um bancário que, após o adoecimento, não consegue mais entrar em nenhuma agência bancária, mesmo que seja para pagar uma conta ou usar o caixa eletrônico, enfim, para resolver questões cotidianas pessoais; é comum o relato de "não conseguir passar nem na porta de um banco" - qualquer banco, não só aquele do qual é funcionário - sob pena de apresentar intensos sintomas ansiosos. Mesmo após afastamento prolongado das atividades, este comportamento de distanciamento permanece como que "cristalizado", o que, muitas vezes, leva à incapacidade laborativa definitiva.

Esta apresentação é compatível com a característica de Despersonalização/Cinismo e é, a nosso ver, o que diferencia clinicamente os "portadores de burnout" dos pacientes deprimidos em geral.

Dentro de uma perspectiva clínica, portanto, tal persistência da Despersonalização/Cinismo simplesmente não nos permite ignorá-la enquanto parte integrante do fenômeno burnout. No entanto, a pesquisa empírica tem questionado a sua relevância conceitual.

É interessante notar que, em contraposição à dimensão Exaustão (mais facilmente identificável, por ter como característica definidora a fadiga, que tem uma expressão "física”, além do componente mental), a Despersonalização/Cinismo pode ser considerada uma dimensão mais propriamente "subjetiva" do burnout, na medida em que representa uma atitude "negativa” baseada em um sentimento de desencanto, que 
por sua vez é dirigido especificamente ao trabalho. Ou seja, a Despersonalização parece ser, essencialmente, uma manifestação da subjetividade individual. Talvez daí provenha inclusive certa dificuldade, ou dissenso, entre os pesquisadores em nomeá-la, como evidenciado tanto pelas várias proposições para sua "rotulação" (Despersonalização, Cinismo, Desengajamento), quanto pela multiplicidade de manifestações que podem ser consideradas características desta dimensão, desde a indiferença, passando pela irritabilidade, até a evitação (GARDEN, 1987; SHIROM, 2003).

O que nos faz pensar: "Seria o questionamento científico a respeito da natureza da Despersonalização o reflexo de uma dificuldade (uma limitação) do método empírico em avaliar características subjetivas?"

Em caso afirmativo, voltar nossa atenção à clínica, aos casos reais, poderia ajudar a preencher esta lacuna do conhecimento sobre o burnout. Trata-se de enfocar o indivíduo do ponto de vista simbólico (de maneira complementar à perspectiva individual orgânica, fisiológica). Tal abordagem poderia auxiliar, por exemplo, na elaboração de terapias mais eficazes para o burnout - ou, melhor dizendo, para os indivíduos com burnout. Atualmente, a maior parte dos estudos sobre seu tratamento e prevenção prioriza a implementação de estratégias de "manejo de estresse”, em grande parte baseadas em psicoterapia cognitiva, e cujo principal resultado é a redução dos níveis de exaustão (VAN DIERENDONCK; SCHAUFELI; BUUNK, 1998; HÄTINEN et al., 2004). As taxas de retorno ao trabalho a longo prazo, no entanto, permanecem quase que inalteradas (GROSSI; SANTELL, 2009).

\section{Conclusão}

Neste artigo procuramos dar uma ideia da diversidade teórica que forma o campo de pesquisa em burnout, tomando como exemplo alguns aspectos relativos à sua discussão conceitual. Vimos que o burnout não é um conceito "fechado", apesar de haver uma definição dominante; uma importante razão para este domínio foi o desenvolvimento de um instrumento capaz de mensurá-lo quantitativamente, permitindo assim sua investigação epidemiológica.

O debate a respeito do conceito de burnout traduz o desejo de se compreender a sua "verdadeira natureza”, a sua "essência”. Essa tentativa de compreensão tem sido empreendida por meio da investigação científica baseada no método empírico. Curiosamente, as questões que emergem deste debate conceitual (se burnout é só exaustão, se a Despersonalização faz parte do conceito, se se trata de um tipo de resposta de estresse não necessariamente ligada ao trabalho) são justamente as questões nas quais a subjetividade está mais presente. É possível que elas reflitam uma limitação do método empírico (já que a sua ênfase está em descobrir os dados objetivos).
Esta possível limitação teria a ver com as especificidades de um modelo científico da etiologia de doenças - modelo que vem a ser o preferencialmente adotado pela Psiquiatria enquanto ramo da Medicina - fundamentado no princípio de que as doenças existem por si só, independentemente de serem nomeadas (BOGENSCHUTZ; NURNBERG, 2000). Dentro deste modelo, o status de "doença" só é alcançado quando se conhecem os mecanismos biológicos que estariam "na base" (portanto, como causas) da doença em questão (BOGENSCHUTZ; NURNBERG, 2000). Ou seja, o modelo científico atualmente adotado pela Psiquiatria (como disciplina médica) é o de uma etiologia biológica das doenças (KENDELL; JABLENSKY, 2003).

A pesquisa empírica, sendo o método de investigação que serve a tal referencial teórico, tenta (de modo geral e, portanto, também no caso do burnout) estabelecer a validade de um constructo por meio da realização de estudos epidemiológicos. Logo, na medida em que não é possível evidenciar de forma positiva as bases biológicas do burnout, este fenômeno não poderia ser considerado uma "doença”, de acordo com este modelo teórico. É com base neste argumento que alguns autores questionam inclusive a pertinência de se proceder à investigação da validade diagnóstica do burnout, alegando que este é um conceito "inventado", não representativo, de uma "doença real” (MILLAN, 2007; FERRADA-NOLI apud FRIBERG, 2009).

Porém, mesmo que o burnout não possa (e nunca venha a poder) ser considerado uma doença do ponto de vista do modelo científico vigente, isto em nada diminui a importância de estudá-lo. Nem do ponto de vista social (tanto devido às suas causas, relacionadas primariamente à organização do trabalho, quanto por suas inquestionáveis consequências em nível socioeconômico), nem do individual.

Dentro de uma perspectiva clínica, a compreensão do fenômeno individual é, talvez, o que mais interessa. E esta compreensão passa pela consideração dos fatores subjetivos envolvidos na manifestação desse tipo de sofrimento psíquico. Com base na experiência clínica, torna-se impossível ignorar a magnitude destes fatores subjetivos. É somente a partir deles que se pode pensar em um tratamento para as pessoas com quadros de burnout.

E é aí que reside a especificidade da Psiquiatria enquanto campo de saber que inclui a experiência subjetiva do adoecimento como objeto de investigação. Mas trata-se, antes de mais nada, de ampliar o escopo desta disciplina para além do enfoque atual, que prioriza a busca de correlatos biológicos para o sofrimento psíquico. Através do estudo do burnout, a Psiquiatria poderia oferecer uma contribuição singular ao campo da Saúde Mental e Trabalho e em condições de ocupar, inclusive, uma posição privilegiada neste estudo ao permitir a integração das perspectivas física/fisiológica, psicológica e social na compreensão dos fenômenos humanos. 


\section{Referências}

AHOLA, K. et al. The relationship between jobrelated burnout and depressive disorders: results from the Finnish Health 2000 Study. Journal of Affective Disorders, v. 88, p. 55-62, 2005.

. Burnout in the general population: results from the Finnish Health 2000 Study. Social Psychiatry and Psychiatric Epidemiology, v. 41, n. 1, p. 11-17, 2006a.

. Alcohol dependence in relation to burnout among the Finnish working population. Addiction, v. 101, n. 1, p. 1438-1443, 2006b.

BOGENSCHUTZ, M. P.; NURNBERG, H. G. Classification of mental disorders. In: SADOCK, B. J.; SADOCK, V. A. Kaplan and Sadock's comprehensive textbook of psychiatry. 7. ed. Philadelphia, PA: Lippincott Williams \& Wilkins, 2000. p. 824-853.

CARLOTTO, M. S.; PALAZZO, L. S. Síndrome de burnout e fatores associados: um estudo epidemiológico com professores. Cadernos de Saúde Pública, v. 22, n. 5, p. 1017-1026, 2006.

COX, T.; TISSERAND, M.; TARIS, T. The conceptualization and measurement of burnout: questions and directions (Editorial). Work \& Stress, v. 19, n. 3, p. 187-191, 2005.

DEMEROUTI, E. et al. From mental strain to burnout. European Journal of Work and Organizational Psychology, v. 11, n. 4, p. 423-441, 2002a.

. The convergent validity of two burnout instruments: a multitrait-multimethod analysis. European Journal of Psychological Assessment, v. 18, n. 3, p. 296-307, 2002b.

ENZMANN, D. et al. Dimensionality and validity of the Burnout Measure. Journal of Occupational and Organizational Psychology, v. 71, p. 331-352, 1998.

FREUDENBERGER, H. J. Staff burnout. Journal of Social Issues, v. 30, p. 159-165, 1974.

FREUDENBERGER, H. J.; RICHELSON, G. L'Épuisement professionel: la brûlure interne. Tradução Marc Pelletier. Ottawa, ON: Gaëtan Morin, 1987.

FRIBERG, T. Burnout: from popular culture to psychiatric diagnosis in Sweden. Culture, Medicine and Psychiatry, v. 33, p. 538-558, 2009.

GARDEN, A. M. Depersonalization: a valid dimension of burnout? Human relations, v. 40, p. 545-560, 1987.

GROSSI, G. et al. The morning salivary cortisol response in burnout. Journal of Psychosomatic Research, v. 59, p. 103-111, 2005.

GROSSI, G.; SANTELL, B. Quasi-experimental evaluation of a stress management programme for female county and municipal employees on longterm sick leave due to work-related psychological complaints. Journal of Rehabilitation Medicine, v. 41, p. 632-638, 2009.

HALBESLEBEN, J. R. B.; DEMEROUTI, E. The construct validity of an alternative measure of burnout: investigating the english translation of the Oldenburg Burnout Inventory. Work \& Stress, v. 19, n. 3, p. 208-220, 2005.

HALLSTEN, L. Burnout and wornout: concepts and data from a national survey. In: ANTONIOU, A. S. G.; COOPER, C. L. (Ed.). Research companion to organizational health psychology. Cheltenham, UK: Elgar, 2005. p. 516-536.

HÄTINEN, M. et al. Burnout patterns in rehabilitation: short-term changes in job conditions, personal resources, and health. Journal of Occupational Health Psychology, v. 9, n. 3, p. 220-237, 2004.

HOBFOLL, S. E.; SHIROM, A. Stress and burnout in work organizations. In: GOLEMBIEWSKI, R. T. (Ed.). Handbook of organization behavior. New York: Dekker, 1993. p. 41-61.

HONKONEN, T. et al. The association between burnout and physical illness in the general population: results from the Finnish Health 2000 Study. Journal of Psychosomatic Research, v. 61, p. 59-66, 2006.

KALIMO, R. The challenge of changing work and stress for human resources. The case of Finland. Journal of Tokyo Medical University, v. 58, n. 3, p. 349356, 2000.

KENDELL, R.; JABLENSKY, A. Distinguishing between the validity and utility of psychiatric diagnoses. American Journal of Psychiatry, v. 160, p. 4-12, 2003.

KRISTENSEN, T. S. et al. The Copenhagen burnout inventory: a new tool for the assessment of burnout. Work \& Stress, v. 19, n. 3, p. 192-207, 2005.

LEHRMAN, Y. et al. Association between burnout at work and leukocyte adhesiveness/aggregation. Psychosomatic Medicine, v. 61, p. 828-833, 1999.

LEITER, M. P. Conceptual implications of two models of burnout. Group \& Organization Studies, v. 14, n. 1, p. 15-22, 1989

MASLACH, C.; JACKSON, S. E.; LEITER, M. P. Maslach burnout inventory manual. 3. ed. Palo Alto, CA: Consulting Psychologist's Press, 1996.

. Maslach burnout inventory. In: ZALAQUETT, C.; WOOD, R. J. (Ed.). Evaluating stress: a book of resources. Lanham, MD: Scarecrow Press, 1997. p. 191-218.

MASLACH, C.; SCHAUFELI, W. B.; LEITER, M. P. Job burnout. Annual Review of Psychology, v. 52, p. 397422, 2001.

MELAMED, S. et al. Burnout and risk of cardiovascular disease: evidence, possible 
causal paths, and promising research directions. Psychological Bulletin, v. 132, n. 3, p. 327-353, $2006 a$.

Burnout and risk of type 2 diabetes: a prospective study of apparently healthy employed persons. Psychosomatic Medicine, v. 68, p. 863-869, 2006b.

MILLAN, L. R. A síndrome de burnout: realidade ou ficção? Revista da Associação Médica Brasileira, v. 53, n. 1, p. 5, 2007.

MOCH, S. L. et al. Longitudinal changes in pituitaryadrenal hormones in South African women with burnout. Endocrine, v. 21, n. 3, p. 267-272, 2003.

MOMMERSTEEG, P. M. C. et al. A longitudinal study on cortisol and complaint reduction in burnout. Psychoneuroendocrinology, v. 31, p. 793-804, 2006.

PARKER, P. A.; KULIK, J. A. Burnout, self- and supervisor-rated job performance, and absenteeism among nurses. Journal of Behavioral Medicine, v. 18, n. 6, p. 581-599, 1995.

PINES, A. M.; ARONSON, E. Burnout: from tedium to personal growth. New York: Free Press, 1981.

PINES, A. M.; KEINAN, G. Stress and burnout: the significant difference. Personality and Individual Differences, v. 39, n. 3, p. 625-635, 2005.

SCHAUFELI, W. B.; ENZMANN, D. The burnout companion to study and practice: a critical analysis. London: Taylor and Francis, 1998.

SCHAUFELI, W. B. et al. On the clinical validity of the Maslach burnout inventory and the burnout measure. Psychology \& Health, v. 16, p. 565-582, 2001.

SCHAUFELI, W. B.; TARIS, T. The conceptualization and measurement of burnout: common grounds and worlds apart. Work \& Stress, v. 19, n. 3, p. 256-262, 2005.

SHIROM, A. Burnout in work organizations. In: COOPER, C. L.; ROBERTSON, I. (Ed.). International Review of Industrial and Organizational Psychology. New York: Wiley, 1989. p. 26-48.
. Job-related burnout: a review. In: QUICK, J. C.; TETRICK, L. E. (Ed.). Handbook of occupational health psychology. Washington, DC: American Psychological Association, 2003. p. 245-265.

SHIROM, A. et al. Effects of work overload and burnout on cholesterol and triglycerides levels: the moderating effects of emotional reactivity among male and female employees. Journal of Occupational Health Psychology, v. 2, n. 4, p. 275-288, 1997.

. Burnout, mental and physical health: a review of the evidence and a proposed explanatory model. International Review of Industrial and Organizational Psychology, v. 20, p. 269-309, 2005.

SILVEIRA, N. M. et al. Avaliação de burnout em uma amostra de policiais civis. Revista de Psiquiatria do Rio Grande do Sul, v. 27, n. 2, p. 159-163, 2005.

SONNENTAG, S. Burnout research: adding an offwork and day-level perspective. Work \& Stress, v. 19, n. 3, p. 271-275, 2005.

TOPPINEN-TANNER, S. et al. Burnout as a predictor of medically certified sick-leave absences and their diagnosed causes. Behavioral Medicine, v. 31, n. 1, p. 18-27, 2005.

TUCUNDUVA, L. T. C. M. et al. A síndrome da estafa profissional em médicos cancerologistas brasileiros. Revista da Associação Médica Brasileira, v. 52, n. 2, p. 108-112, 2006.

VAN DIERENDONCK, D.; SCHAUFELI, W. B.; BUUNK, B. P. The evaluation of an individual burnout intervention program: the role of inequity and social support. Journal of Applied Psychology, v. 83, p. 392407, 1998.

WEBER, A.; JAEKEL-REINHARD, A. Burnout syndrome: a disease of modern societies? Occupational Medicine, v. 50, n. 7, p. 512-517, 2000.

WEBER, A.; WELTLE, D.; LEDERER, P. Ill health and early retirement among school principals in Bavaria. International Archives of Occupational and Environmental Health, v. 78, p. 325-331, 2005. 